\title{
When is surgery research? Towards an operational definition of human research
}

Curtis E Margo Watson Clinic, Lakeland, Florida, USA

\begin{abstract}
The distinction between clinical practice and surgical research may seem trivial, but this distinction can become a complex issue when innovative surgeries are substituted for standard care without patient knowledge. Neither the novelty nor the risk of a new surgical procedure adequately defines surgical research. Some institutions tacitly allow the use of new surgical procedures in series of patients without informing individuals that they are participating in a scientific study, as long as no written protocol or hypothesis exists. Institutions can justify this practice by viewing human research in narrow terms as an activity outlined in a formal protocol. Application of limited definitions, however, erodes patients' rights and risks losing public confidence in how biomedical research is conducted. I propose an operational definition of human research also be recognised. Enforcing more rigid and less ambiguous guidelines of human research may curtail enrolment into some studies, but it will also protect patients from being used as subjects without their knowledge.

(Fournal of Medical Ethics 2001;27:40-43)
\end{abstract}

Keywords: Experimental surgery; innovative surgery; medical ethics; surgical research

"... the process of protecting human study subjects essentially begins with a process of prospective selfreferral by investigators of themselves and their research to IRBs. .." Gary B Ellis ${ }^{1}$

A number of years ago I discovered that a colleague was conducting what seemed to be a clinical trial to determine whether by adapting an already accepted surgical procedure, he could improve upon its results by operating at an earlier stage in the disease process. The rationale and scientific justification for the study were understandable. The study was not randomised. A series of patients was gathered from his clinical practice. Patients with this disorder who met certain clinical criteria were enrolled. Outcome was assessed by the usual surrogate measures obtained during postoperative follow up examinations. Preliminary data from the trial were presented at a professional meeting. Issues about safety and effectiveness were inconclusive so more patients were added to the study until it became apparent that the risk of surgical complication outweighed any potential benefit of early treatment. Innovation through deviation from standard practice is an important means of improving surgical care and needs to be encouraged even if results may not always turn out as expected. What troubled me about this particular case, however, was that the patients were neither informed about being entered into a study nor told about the research hypothesis, nor had the study been submitted to the scrutiny of an institutional review board (IRB).

Since the time of that study, I have known about similar trials and heard about others. I do not think they are a problem unique to the institutions I have been associated with. Discussion with other physicians concerning this type of research has confirmed my impression that this is a widespread practice and a subject that can invoke several divergent interpretations. Many view the process as within the scope of clinical practice at an academic medical centre and not as research at all. Others consider it human experimentation and are bothered by the lack of disclosure and the absence of informed consent.

These types of interventional studies have several characteristic features: 1 ) they consist of a series of patients; 2) outcome measures are common clinical parameters, the type usually obtained during routine clinical follow up; 3 ) effectiveness is determined by comparison with historical controls; 4) formal written protocols do not exist, and 5) because these activities are viewed as clinical care, they are invisible to institiutional review boards. Studies that show some potential may be presented at scientific meetings, reported in none-peer-review journals, prepared for abstract, or submitted for publication as a "retrospective study", "pilot study", "case series", or "observational study". Although there is no collective title given to these types of studies, terms often used to describe their conceptual framework are informal research, hypothesis exploring data collection, preliminary clinical work, or informal data collection.

For the purpose of this discussion, this concept will be referred to as informal research. From my perspective, informal research is used to test surgical procedures. This may be because of the variety of difficulties associated with planning randomised surgical trials, or perhaps because scientists simply demand more rigorous proof of clinical effectiveness for drugs than for surgery. Informal research does not include the mindful surgeon who monitors patient outcomes to detect deficiencies or to identify areas for possible improvement. This type of self-imposed audit is not testing a scientific hypothesis. 
The debate over whether these studies are research or clinical practice is contentious and threatening. ${ }^{2-5}$ Any professional discussion about the ethical implications of conducting informal research not only impugns the character of those involved, it also raises vexing legal and financial questions. The prevalence of informal research is unknown. An investigation by the University of South Florida Standing Committee on Research Misconduct found that informal research is a normative practice in some academic settings. ${ }^{6}$ This conclusion was based on a thorough review of human research prompted by the case of a surgeon who, at regular intervals, analysed the postoperative results of patients who underwent an innovative surgical procedure. Patients were not told about the innovative nature of the surgery. Clinical data were used to update abstracts, presentations and material for multiple publications for two years. ${ }^{7}$ The study was described as "retrospective" and "observational", and the methodology was called "informal data collection". Because there was no written protocol and the study did not involve randomisation, the standing committee on research misconduct concluded that the activity was clinical practice consistent with federal guidelines on human research ${ }^{8}$

Although the distinction between normal surgical variation and innovation can be ill-defined, the difference should be apparent in certain situations. The application for a patent on a surgical procedure, for instance, would indicate that the procedure departs significantly from standard practice. If a surgical procedure is described by the surgeon at meetings or to the public as "new", "innovative", or "a major breakthrough", it can only be interpreted as a departure from normal procedure. Another indication that a surgical procedure deviates substantially from pre-existent procedures is the need to "refine" the technique in the animal laboratory before introducing it into the operating room.

The increasing emphasis on technological innovation and the practice of describing "new" surgeries to the newspapers before they are formally tested, makes the need for more thoughtful dialogue about what constitutes human research and how to best protect patients' rights even more urgent. I will propose a simple and understandable definition of human research that may make it more difficult for clinical scientists to disguise prolonged, informal studies as clinical care.

\section{New surgical procedures}

The medical profession has largely ignored the ethical tensions associated with the introduction of new surgeries and the transfer of surgical procedures from the laboratory to the operating room. In this area of experimental therapeutics, both the patient and the patient-physician relationship are vulnerable. The most likely threat to patients may not be that an untested treatment is unsafe, but that the trust of patients in their physicians may be compromised by the goals and pressures of research.

The fact that a procedure is experimental, new or untested does not necessarily mean its use is research. When a procedure departs substantially from the standard of care, however, it should be studied for safety and effectiveness at the earliest time possible. Because there are no guidelines which define threshold levels of clinical innovation or acceptable added risk, the responsibility for judging when a new or novel clinical practice becomes research rests solely with the individual surgeon. ${ }^{19}$ Unlike drugs, medical devices and other products that require demonstration of safety and effectiveness before approval by the Food and Drug Administration, there are no regulations describing how new surgical procedures should be tested. ${ }^{10}$ Currently there is greater oversight protection in place for laboratory animals than there is for testing innovative surgeries in humans.

\section{Research, clinical practice and informal research}

The distinction between clinical practice and research can be blurred. The problem with this overlap is most obvious at academic medical centres where the potential rewards for successful research are the greatest. The National Commission for the Protection of Human Subjects of Biomedical and Behavioral Research offers some guidance by defining the term "research" as "an activity designed to test an hypothesis, permit conclusions to be drawn, and thereby to develop or contribute to generalizable knowledge". ${ }^{11}$ The commission also states: "Research is usually described in a formal protocol that sets forth an objective and a set of procedures designed to reach that objective. ${ }^{11}$

By comparison, clinical practice is an activity that involves undivided commitment to patient wellbeing. Duty to patients is not diluted by other goals. Yet physicians in clinical practice have always learned from their patients. Based on professional knowledge and experience, physicians will seek creative solutions to clinical problems. Serious illnesses or diseases with poor prognoses may require innovative therapies that involve considerable risk, so neither the novelty of a procedure nor its potential risk are especially useful in distinguishing clinical practice from research. Therapeutic success in individual patients who have undergone an innovative procedure will naturally lead to thoughts that it may benefit an entire population of patients with the same disease. An interventional case series intended to test such a thought can easily go unrecognised as clinical research unless a physician takes the initiative to formalise the study or the data are formally presented or published.

The origins of informal research are speculative, but its underpinnings may be a remnant of the paternalistic culture that existed in medicine after the second world war when the norms and practice of medicine were quite different. The authority physicians gave themselves just 40 to 50 years ago to be the best and often only judge of what patients 
should and should not know about clinical experiments stands in contrast to current ethical norms. ${ }^{12}$ The medical profession now appreciates the primary role patients have in determining their care, but informal research has the ethos of an earlier era in medicine when patients were not involved in clinical decision making.

The concept of informal research has persisted because it helps to deal with awkward transitiona activities that look like human research-but officially are not. Informal research also serves a practical role by weeding out bad ideas without much fanfare and by getting good ideas to a stage where they can be formally tested. Randomised clinical trials have created a conspicuous hiatus between the development of a surgical procedure in the laboratory and its definitive (ie formal) testing in humans. This gap has largely been filled by informal research. Innovative surgical procedures that have been developed in animal models simply cannot go from the laboratory to randomised trials without the refinements and modifications made possible by testing in human subjects. ${ }^{1314}$ Although the preliminary stages of surgical research in humans can be instituted under formal protocol (and many are), there are no administrative mechanisms and no strong moral will to prevent these studies from being disguised as clinical practice.

\section{An operational definition of human research}

The medical profession may be reluctant to pursue the problems associated with informal surgical research for several reasons, including possible negative repercussions related to third party payers. ${ }^{15}$ When asked to determine the boundaries of human research, academic medical centres in the United States can defer to federal guidelines that view human research as a process outlined in a formal document. ${ }^{16}$ These guidelines are inadequate because they fail to consider that human research can exist in the absence of a written protocol. One challenge in protecting patient autonomy is to be able to identify the process of research when no formal protocol exists. I submit that this involves establishing criteria for informal research that are operational in nature and that correspond to intent.

The critical question that must be addressed in an interventional case series is at what point in time does clinical practice become research. The defining elements of research are a scientific hypothesis and the systematic collection of data to support or disprove the hypothesis. These elements are essential features of research, no matter how good or how bad the methodology may be. The implicit assumption in a surgical case series performed as informal research, however, is that the clinical hypothesis was not formalised until after the therapeutic intervention.

The time of data collection is not a reliable or sensitive means to determine intent to perform research. The information collected for clinical care is essentially the same as that for informal research.
It can be harvested for analysis at any time during follow up.

While it is difficult, if not impossible, to stipulate just when observational results of an innovative surgical treatment become scientific research, that boundary is certainly crossed once a report is presented at a scientific meeting or submitted for scientific review. The essential elements of research, even if preliminary, now exist together. After a case series has been formally presented or submitted for publication, there can be no turning back. Any addition of new patients to enlarge an existing clinical database is a clear departure from just clinical practice to clinical research. After a scientific hypothesis is a matter of record, the physician who enters more patients into the same series needs to regard the work as research as well as himself or herself as a researcher.

\section{The loss of public confidence}

It is possible that too stringent a definition of human research will deter physicians from offering therapies of unproven benefit even though they may be in the best interest of the patient. Also, strict guidelines may stifle needed innovation and progress. On the other hand, if the medical profession includes all informal research under the umbrella of clinical practice, we risk losing public confidence in how biomedical research is conducted. In the United Sates and elsewhere, a gap exists between the formal federal definition of human research and what the public perceives as human experiments. ${ }^{17}$ There is a point when the application of too limited a definition of human research works against common sense and threatens credibility.

The public may also sense monetary reasons behind the disguising of surgical trials as clinical care. The fear that formal preliminary testing of innovative surgeries might not be reimbursed by third party payers may be a strong disincentive for academic medical centres to police seriously the area of informal research.

\section{Bad science}

Informal research by its very nature is a flawed process. Studies of new surgical procedures in which there is no prespecified hypothesis are prone to bias. Valid conclusions regarding the effectiveness of new therapies can seldom be drawn from studies without adequate controls. Informal research not only undermines the patient-physician relationship, it has the potential to pollute the medical literature and news media with what is in fact "bad science". ${ }^{18}$

This is not to say, however, that early "formal" studies on surgical procedures are going to be definitive. They will not be. Like results from phase 1 and phase 2 drug studies, promising but preliminary results will need to undergo more rigorous testing. Although preliminary surgical studies are imperfect, they need to be conducted openly and not disguised as clinical care then later reported as a retrospective series. 


\section{Conclusions}

It is the surgeon's ethical and legal prerogative to use innovative surgery as treatment, but when the results of new surgical procedures are collected, analysed and submitted as evidence of safety and effectiveness the surgery itself must be considered as being part of a research project. Although the division between clinical practice and research will always be imperfect, current safeguards for subjects of human research can be improved. Awareness of the moral hazards associated with testing innovative surgeries in non-randomised trials may be enhanced by more clearly defining the elements of human research and by acknowledging that research can exist in the absence of a written protocol.

The United States Advisory Committee on Human Radiation Experiments recommended that the "medical profession intensify its commitment to the ethics of research involving human subjects". ${ }^{12}$ This recommendation also applies to the transfer of new surgical procedures from the animal laboratory to the operating room. We should protect subjects of human research by more clearly articulating guiding principles of conduct for dealing with patients enrolled in non-randomised clinical trials. This goal will not be met unless we affirm the primacy of patients' rights and do not subordinate these rights for the "greater good" of scientific investigation. ${ }^{190}$

Curtis Margo, MD, MPH, is an Ophthalmologist, a Pathologist and a Partner in the Watson Clinic LLP, Lakeland, Florida USA.

\section{References and notes}

1 Ellis GB. Keeping research subjects out of harm's way. Fournal of the American Medical Association 1999;282:1963-5.
2 Lyon J. Experimenting with humans. Part 2: innovation or exploitation? Second Opinion 1988;8:10-42.

3 Dossetor JB. Innovative treatment versus clinical research: an ethics issue in transplantation. Transplantation Proceedings 1990;22:966-8.

4 Ward CM. Surgical research, experimentation and innovation. British fournal of Plastic Surgery 1994;47:90-4.

5 Anonymous [editorial]. Qualms about innovative surgery. Lancet 1985;1:149.

6 Wolfson J, Bellante D, Sarkar S, Paveza GJ, Lazaridis EN, Updegraff S. The final report of the University of South Florida Standing Committee on Research Misconduct. December 14, 1999 [public document]. Office of General Counsel, University of South Florida, College of Medicine: 24-7.

7 See reference 6: 15-16; 23-4.

8 See reference 6: 22-4.

9 Love J. Drugs and operations. Some important differences. fournal of the American Medical Association 1975;232:37-8.

10 Spodick DH. Numerators without denominators: there is no FDA for the surgeon. Fournal of the American Medical Association 1975;232:35-6.

11 Belmont Report. Ethical principles and guidelines for the protection of human subjects of research. Federal Register 1979;44: 23191-7. Those who have difficulties tracing this material through the Federal Register can find the Belmont Report via: Washington DC: DHEW, 1978. Publication No (OS) 78-0012.

12 Research ethics and the medical profession. Report of the Advisory Committee on Human Radiation Experiments. FourAdvisory Committee on Human Radiation Experiments.
nal of the American Medical Association 1996;276:403-9.

13 Bonchek LI. Are randomized surgical trials appropriate for evaluating new operations? New England Fournal of Medicine 1979;301:44-5.

14 Bunker JP, Hinkley D, McDermott WV. Surgical innovation and its evaluation. Science 1978;200:937-41.

15 Gleeson S. Paying for innovations in surgery. Bulletin of the American College of Surgeons 1996;81:10-3.

16 See reference 6: 22 .

17 Charo RA. Human subjects have it worse than guinea pigs. Chronicle of Higher Education 1999: Jun 25: A64.

18 Waring GO III. A cautionary tale of innovation in refractive surgery. Archives of Ophthalmology 1999;117:1069-73.

19 Bulger RE. Toward a statement of the principles underlying responsible conduct in biomedical research. Academic Medicine 1994;69:102-7.

20 Woodward B. Challenge to human subject protection in US medical research. Fournal of the American Medical Association 1999;282:1947-52. 